\title{
The feature-positive effect and sign-tracking behavior during discrimination learning in the rat
}

\author{
CHARLES R. CROWELL and THOMAS P. BERNHARDT \\ University of Notre Dame, Notre Dame, Indiana 46556
}

\begin{abstract}
Feature-positive (FP) and feature-negative (FN) successive discrimination learning was investigated in the rat. When a discrete, visual element serving to differentiate the discriminanda belonged to $S+(F P)$, rats acquired the discrimination more rapidly than when it formed a part of $S-$ (FN). During the course of training, FP rats developed a tendency to direct their responding toward the differentiating feature in $\mathrm{S}+$ while $\mathrm{FN}$ rats shifted their responding away from this element in S-. These findings were discussed in terms of the conceptions of stimulus-reinforcer relations and "sign-tracking" behavior.
\end{abstract}

Jenkins and Sainsbury $(1969,1970)$ investigated successive discrimination learning in the pigeon using paradigms in which the positive $(S+)$ and negative $(\mathrm{S}-)$ stimulus displays were composed of discrete visual elements. Some elements were common to both displays, but one feature differentiated the discriminanda. In the feature-positive (FP) arrangement, the distinguishing feature was embedded in $\mathrm{S}+$ among the common elements. In the other, featurenegative (FN) paradigm, the differentiating feature was located among common elements in S-. Comparison of the rates of acquisition of the successive discrimination under these two arrangements revealed that FP subjects ceased responding on $\mathrm{S}-$ trials within a few sessions while FN birds exhibited little evidence of such learning even after extended training. The marked superiority of acquisition under FP conditions represents a differential effect of these two training procedures that has come to be known as the "feature-positive effect."

Another disparate consequence of FP and FN training observed by Jenkins and Sainsbury concerned the reaction of subjects to the differentiating feature in the stimulus displays. In these studies, each element of both discriminative stimuli was superimposed on a separate response key, which allowed pecking directed at all elements of $S+$ and $S-$ to be monitored throughout training. The tendency for FP birds to peck at the distinguishing feature within $\mathrm{S}+$ was

Portions of this study were presented at the convention of the Midwestern Psychological Association, Chicago, 1976. We are grateful to S. Manganello, J. V. Lupo, W. Moredock, R. Gilpatrick, and P. Kubiak for their assistance in various stages of this research. The second author is now at the computer services division of the Associates Corporation, South Bend, Indiana. Requests for reprints should be sent to Dr. Charles R. Crowell, Department of Psychology, University of Notre Dame, Notre Dame, Indiana 46556. observed to increase rapidly during initial sessions, until virtually all responding on positive trials was directed there. In contrast, FN birds learned to direct nearly all their responding away from the distinguishing element in $\mathrm{S}-$, toward the elements common to both discriminanda. This second differential effect of FP and FN training is of particular interest because there was no obvious differential reinforcement in either paradigm for responding directed at the feature differentiating the stimulus displays. Reinforcement was contingent upon responding during positive trials in both paradigms, but the location of pecking to $\mathrm{S}+$ was irrelevant. Similarly, responding anywhere on the $S$ - display was accompanied by nonreward in both training procedures. Therefore, changes in the distribution of pecks directed at the various elements in the discriminanda were observed in the absence of explicit contingencies of reinforcement that would promote such effects. Moreover, it is unlikely that these changes in responding were produced by adventitious reinforcement, in view of the results of a study indicating that such effects occur even when accidental reinforcement is precluded (Jenkins, 1973).

Hearst (1975) and Hearst and Jenkins (1974) have noted a possible connection between an element's apparent food-signaling function and the spatial redirection of responding that may occur with respect to it during FP and FN training. In an FP condition, the distinguishing feature within $\mathrm{S}+$ may be viewed as the most "positive predictor" of food inasmuch as it has the highest positive correlation with delivery of the reinforcer. Furthermore, it is the locus of redirected responding on $\mathrm{S}+$ trials. With an FN arrangement, the differentiating feature in $\mathrm{S}-$ is best characterized as a "negative predictor" of food, since it bears an inverse relation to the occurrence of the reinforcer. In this context, elements 
common to both stimulus displays have the highest positive relation to food and are observed to provide the target for responding that comes to be directed away from the distinguishing feature. For Hearst and Jenkins, these circumstances indicate that an organism's behavior is importantly guided by prevalent stimulus-reinforcer relations, such that "positive predictors" of food come to be approached and contacted, whereas signals of nonreward come to be avoided. These authors have characterized such stimulus-reinforcer based directed action as "signtracking"' behavior (Hearst and Jenkins, 1974).

The literature on the feature-positive effect (see Hearst, 1978, for a review) indicates that while most studies of this phenomenon have employed the pigeon, at least two have obtained a similar finding during appetitive discrimination learning in the rat (Halgren, 1974; Lea, 1974). Unfortunately, however, neither included an assessment of possible signtracking tendencies that may have emerged during the course of training. The purpose of the present experiment was to replicate these previous findings with the rat, and also to determine whether responding comes to be directed toward or away from certain elements of the stimulus displays during FP and FN discrimination training in this species. The overall plan of the study involved a comparison of the performance of groups exposed to either an FP or FN successive discrimination arrangement of discrete, visual elements that were spatially localizable.

\section{METHOD}

\section{Subjects}

Twenty naive, male Sprague-Dawley rats between 325 and $375 \mathrm{~g}$ were obtained from the Charles Rivers Company in Wilmington, Massachusetts. They were maintained in individual cages under a 12-h-light/dark cycle, with free access to water throughout the experiment.

\footnotetext{
Apparatus

The experimental chamber for this study was a rectangular box with inside dimensions measuring $23 \mathrm{~cm}$ from side to side, $32 \mathrm{~cm}$ from front to back, and $27 \mathrm{~cm}$ from floor to ceiling. The front wall and top were composed of Plexiglas, the sides and back of aluminum, and the floor of hardware cloth. Three rectangular openings, $3.5 \times 3.8 \mathrm{~cm}$, were cut in one side wall $3 \mathrm{~cm}$ apart and $2 \mathrm{~cm}$ above the floor, the innermost being located $4 \mathrm{~cm}$ from the back wall. Response keys just large enough to cover each reactangular opening were mounted on the outside surface of the box. Each key consisted of a strip of thin translucent milk-white plastic hinged at the top and so positioned that a weight of approximately $7 \mathrm{~g}$ at the bottom would move the plastic through a distance of $2 \mathrm{~mm}$ to activate an adjacent microswitch. Each key was illuminated from behind with one of two intensities of white light produced by separate Lehigh Valley Tri-Color lamps (Model 114-01) mounted directly behind each key. The more intense level was obtained by connecting all three bulbs within each lamp (color caps removed) in parallel to a 26-V power supply. A less intense light was produced by switching a 150 e resistor in series with the three-bulb parallel network. For this study, dimly illuminated keys served as features common to both discriminative stimuli while a single, relatively bright key
}

was used as the differentiating element. A food tray, $8 \mathrm{~cm}$ high, $4.5 \mathrm{~cm}$ wide, and $4 \mathrm{~cm}$ deep, was mounted on the side wall opposite the response keys, directly across from the center key. Access to the tray was provided by a $3.5 \times 3.8 \mathrm{~cm}$ opening in the wall $2 \mathrm{~cm}$ above the floor. The food tray was continuously illuminated from above by a standard $28-\mathrm{V}$ bulb positioned behind a milk-white diffusing screen mounted in the upper portion of the tray. Noyes food pellets $(.045 \mathrm{~g})$ were delivered to the tray from behind the diffusing screen by a Gerbrands pellet dispenser (Model G5100). Programming and recording of events was accomplished by a Digital Equipment Corporation PDP-8/I computer located in another room.

\section{Procedure}

For 4 days following their arrival from the supplier, the rats were allowed free access to food and water in individual cages in order to determine an ad-lib weight level. Each rat was then put on a restricted diet until its weight was stabilized at $85 \%$ of the base-line level for the duration of the experiment.

The first 2 days of the experiment involved magazine training and shaping to press with the nose or paw any one of the three keys, each of which was continuously illuminated with the dim light. No more than 60 reinforcements were allowed in either session. By the 3rd day, stable levels of pressing were obtained and each subject was shifted to a procedure involving 60 discrete trials, with an intertrial interval of $60 \pm 15 \mathrm{sec}$. Each trial consisted of illumination of the three response keys for a period no longer than $20 \mathrm{sec}$. If a single response occurred during this time on any key, the trial was terminated with immediate reinforcement. After $20 \mathrm{sec}$, the trial was terminated automatically without food. Two patterns of lighting the three keys were employed. In one, the uniform display, each key was illuminated with the lower intensity light. The other, referred to below as the feature display, consisted of two dim keys and one relatively bright key. Equal numbers of each display were presented in a semirandom order, with the restriction that no more than three of any one kind could occur in a row. During a feature-display trial, the key location of the feature remained constant, but across all 30 presentations of this display, the bright light appeared on each key equally often according to a random sequence. The procedure on Days 4-8 was identical to that of Day 3 except that the number of responses required to obtain reinforcement during a trial was progressively increased from one to six. From Day 8 on, all trials of each daily session involved an FR-6 response requirement that was fulfilled whenever a total of six responses was distributed on any one or more of the three keys.

On Day 9, two groups of 10 subjects were formed, equated for levels of responding to the bright key in the previous session. Successive-discrimination training was initiated for both groups on this day. For the FP group, FR-6 responding was reinforced only during the feature display (i.e., $\mathrm{S}+$ ). Completion of the ratio requirement during the uniform display (i.e., $S-$ ) was accompanied by trial termination without the food for these subjects. An identical procedure was employed for FN rats except that the roles of the feature and uniform displays were interchanged. Twelve daily sessions of discrimination training was administered to each group. On each trial of these sessions, number of responses to the bright and/or dim keys was recorded.

\section{RESULTS}

From the raw data, a discrimination ratio was computed daily for each subject by dividing the total number of responses to the positive $(\mathrm{S}+)$ display in a session by the total number of responses to both discriminative stimuli. This measure reflects the magnitude of a subject's differential responding to $\mathrm{S}+$ 
and $\mathrm{S}$ - and indicates the extent to which the succesive discrimination has developed. A ratio of .5 indicates that responding occurred with equal frequency on both positive and negative trials, while a ratio of 1.0 indicates that responding was confined exclusively to S + trials. Also, a "tracking ratio" was computed daily based solely upon responding to the feature display. This measure was the proportion of total responding on feature trials in a session that occurred to the differentiating element (i.e., the bright key). This ratio reflects the subject's tendency to follow or track the differentiating element during a series of feature-display trials. If responding were distributed equally among the various elements of the feature display over the sequence of trials in a session, then a ratio of .33 would be obtained. A ratio of 1.0, however, would signify that a subject responded exclusively to the differentiating element on each feature trial, whereas a ratio near 0 would indicate a tendency to avoid responding to the distinguishing feature. In addition to these ratios, daily rates of responding were computed separately for positive and negative trials by dividing the total number of $\mathrm{S}+$ and $\mathrm{S}-$ responses in a session, respectively, by the cumulative duration in seconds of the appropriate type of trial.

The daily mean discrimination and tracking ratios for FP and FN groups are plotted in Figure 1 for the 12 days of discrimination training. It is clear from this graph that the successive discrimination developed progressively in each group during the course of training. Although both appear to have acquired the discrimination to nearly the same extent by Day 12, FP subjects seem to have reached the asymptotic level more rapidly than their FN counterparts. These effects were evaluated statistically by means of a Groups by Days analysis of variance. Both the main effect of Days $[F(11,198)=42.10, p<.05]$ and Groups $[F(1,18)=9.07, p<.05]$, as well as the Days by Groups interaction $[\mathrm{F}(11,198)=2.21$, $\mathrm{p}<.05$ ], were significant. A Newman-Keuls procedure was used for post hoc comparisons of group performance on each day. The mean discrimination ratios of FP and FN groups were significantly different $(p<.05)$ only for Days 6-11. Also, the mean discrimination ratios of both FP $[t(9)=9.9, p<.05]$ and $F N[t(9)=7.79, p<.05]$ groups were significantly higher on Day 12 than on Day 1. Collectively, these tests confirm that while both groups exhibited an improvement in the successive-discrimination to the same terminal level, rats in the FP condition learned more rapidly than those in the FN group.

Also, it can be noted from Figure 1 that the tracking ratios for FP and FN groups changed markedly during the course of discrimination training. For FP subjects, the tendency to follow the distinguishing feature increased rapidly from the initial level to a maximum on Day 4 and then decreased slightly

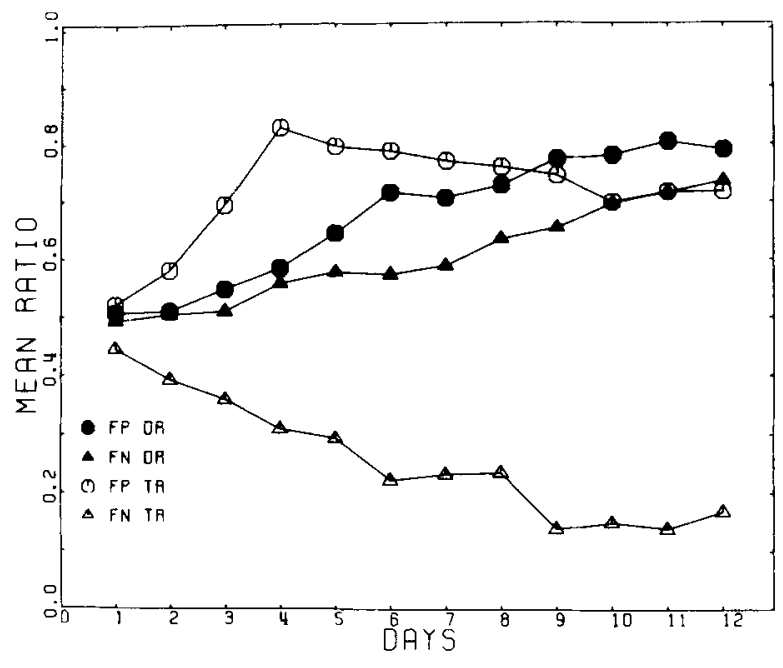

Figure 1. Daily mean discrimination ratios (DR) and tracking ratios (TR) for groups exposed either to a feature-positive (FP) or feature-negative (FN) successive-discrimination schedule.

thereafter to a terminal level still appreciably higher than the initial point. The reliability of this change in responding toward the bright key in $\mathrm{S}+$ was evaluated by means of a trend analysis which revealed that the effect attributable to days had both significant linear $[F(1,99)=6.75, p<.05]$ and significant quadratic $[F(1,99)=23.44, p<.05]$ components. For FN subjects, a tendency to avoid responding to the differentiating element in $\mathrm{S}-$ apparently developed over days. A treatment by subjects analysis of the tracking ratios for this group revealed that the Days effect was highly reliable $[F(11,99)=20.44, p<.05]$. Thus, pronounced changes occurred in the tendency of both groups to respond to the distinguishing element during the course of discrimination training, but in opposite directions for FP and FN subjects.

Figure 2 is a graph of daily response rates for each group on positive and negative trials. Like

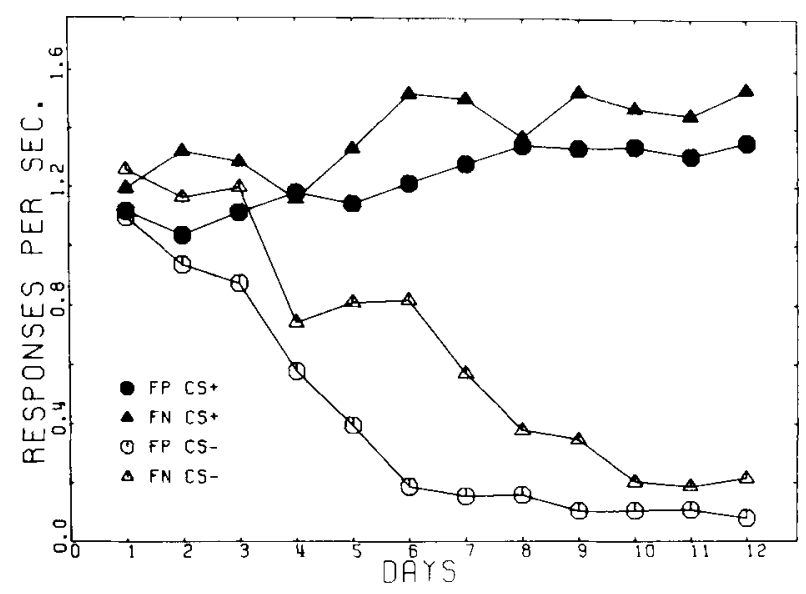

Figure 2. Daily response rates (responses/sec) on positive $(\mathrm{CS}+)$ and negative (CS - ) trials for feature-positive (FP) and feature-negative (FN) groups. 
Figure 1, this graph reveals that both groups gradually developed a tendency to respond differently on S + and $S$ - trials. The figure also shows that the primary difference in the development of the successive discrimination for FP and FN subjects was in the emergence of the ability to withhold responding on negative trials. Response rate data were examined statistically by means of separate Groups by Days analysis of variance applied to the rates obtained from $S+$ and $S-$ trials. The only significant effect from the analysis of positive-trial rates was that attributable to Days $[F(11,198)=3.53, p<.05]$. This result indicates that FP and FN subjects responded similarly on $\mathrm{S}+$ trials and that both exhibited a significant increase in response rate on these trials over the course of training. The analysis of negative-trial data indicated that while both groups showed a general decrease in $\mathrm{S}$ - response rate over the course of training $[F(11,198)=36.33, p<.05]$, the mean $\mathrm{S}$ - response rate over all days for FN subjects was significantly higher than that of FP subjects $[F(1,18)$ $=6.10, \mathrm{p}<.05]$.

\section{DISCUSSION}

An FP arrangement of discriminative stimulus elements was found to be associated with more rapid acquisition of a successive discrimination by rats than was an FN configuration. Moreover, FP superiority was apparent despite noticeable acquisition of differential responding on the part of both FP and FN groups. The general pattern of results is consistent with the results of previous research with the pigeon (cf. Hearst, 1978) and rat (Halgren, 1974), although the magnitude of FN learning here differs from that reported in the original work by Jenkins and Sainsbury.

More importantly, the present findings represent the first demonstration that rats, like pigeons, shift their responding toward and/or away from certain elements of the stimulus displays during the course of FP and FN discrimination learning. Taken together, two aspects of the results support the view that these shifts in responding are examples of signtracking behavior. First, no obvious differential reinforcement can be identified in the present paradigm for spatial redistribution of responding. Like earlier studies, responses to all elements of $\mathrm{S}+$ and $\mathrm{S}-$ contributed equally to the contingency requirements in effect during a particular stimulus period. Second, the direction of the shifts in responding seem to be related to the apparent food-signaling properties of the various elements of the stimulus displays. For FP subjects, responding increased to an element highly correlated with food delivery that differentiated S + and $\mathrm{S}-$. Alternatively, FN rats directed their responding away from a distinguishing feature that consistently signaled nonreward, toward elements partially associated with food. Thus, these findings are consistent with views that emphasize the powerful response-directing effects of stimulus-reinforcer relations (e.g., Hearst \& Jenkins, 1974). Moreover, although an adventitious reinforcement account of these shifts in responding cannot be ruled out on the basis of the present data, existing evidence with rats indicates that sign-tracking tendencies in other situations are not merely by-products of accidental response-reinforcer pairings (Atnip, 1977; Locurto, Terrace, \& Gibbon, 1976; Stiers \& Silberberg, 1974).

The present findings may also have relevance for an interpretation of the feature-positive effect offered by Hearst and Jenkins (1974). These authors have suggested that the successive discrimination may itself be a by-product of the sign-tracking tendencies that emerge in the situation. According to this notion, the sign-tracking behavior of FP subjects would be viewed as a within-stimulus discrimination, based on stimulus-reinforcer relations, that results in the tendencies to respond to the distinguishing features and not to common elements on $\mathrm{S}+$ trials. If the latter tendency to withhold responding generalized to the common elements of $\mathrm{S}-$, then signtracking behavior alone would produce between-trial differential responding appropriate to the discrimination schedule. In support of this hypothesis, the present data indicate that within-stimulus changes in responding for FP subjects tended, on the average, to antedate the occurrence of differential responding indicative of the successive discrimination (see Figure 1). However, this conception leads to the expectation that the sign-tracking tendencies of FN subjects should oppose the development of differential responding to positive and negative stimuli. For these subjects, responding comes to be directed toward the elements common to both $\mathrm{S}+$ and $\mathrm{S}$ trials. Theoretically, therefore, if sign-tracking tendencies were the exclusive determinant of the successive discrimination, no learning under FN conditions would be predicted because common elements, present during $\mathrm{S}+$ and $\mathrm{S}-$, should absorb all responding. The fact that $\mathrm{FN}$ learning occurred in this and other studies (Halgren, 1974; Hearst, 1975; Sainsbury, 1971a) indicates that factors other than stimulus-reinforcer effects no doubt influence the development of such differential reactions. This finding also implies that other factors may support the development of FP discriminations as well.

One factor besides stimulus-reinforcer relations that may have contributed to the development of the successive discriminations in the present study was the differences in overall level of illumination on positive and negative trials. The feature display (with one bright and two dim lights) was characterized by a higher level of nonspecific illumination than the 
uniform display (with only dim lights). Thus, in addition to presence or absence of the differentiating element (one bright key), overall level of illumination may have served as a cue for the differential reaction in both FP and FN paradigms. Although this factor may have contributed to development of the successive discrimination in both the present groups, it is difficult to explain the disparity in their rates of acquisition by this means unless some sort of stimulus dynamism effect were operative. Even in this case, however, it seems unlikely that overall illumination differences could account for the redirected responding that was exhibited under both FP and FN conditions.

Finally, it should be noted that the general conception of sign-tracking behavior described above implies that such tendencies should be observable in any species whenever appropriate stimulusreinforcer relations are present in the environment. This notion has been supported by findings with the pigeon that the presence of certain stimulusreinforcer relations produces directed responding in the context of both feature discrimination (e.g., Jenkins \& Sainsbury, 1969, 1970) and the so-called autoshaping paradigms (e.g., Brown \& Jenkins, 1968). But, although autoshaping has been observed in the rat (e.g., Peterson, 1975; Peterson, Ackil, Frommer, \& Hearst, 1972), sign-tracking in the context of feature-discrimination paradigms has been previously established only for the pigeon (Hearst, 1975; Jenkins \& Sainsbury, 1969, 1970) and the human (Hearst, 1978; Sainsbury, 1971b). Thus, the present findings serve to complement those of the autoshaping studies with rats in demonstrating the species generality of the response-directing effects of stimulus-reinforcer relations.

\section{REFERENCES}

Atnip, G. W. Stimulus- and response-reinforcer contingencies in autoshaping, operant, classical, and omission training procedures in rats. Journal of the Experimental Analysis of Behavior, 1977, 28, 59-69.

Brown, P. L., \& Jenkins, H. M. Auto-shaping of the pigeon's keypeck. Journal of the Experimental Analysis of Behavior, $1968,11,1-8$.
Halgren, C. R. Latent inhibition in rats: Associative or nonassociative? Journal of Comparative and Physiological Psychology, 1974, 86, 74-78.

HEarST, E. Pavlovian conditioning and directed movements. In G. Bower (Ed.), The psychology of learning and motivation (Vol. 9). New York: Academic Press, 1975.

Hearst, E. Stimulus relations and feature selection in learning and behavior. In S. Hulse, H. Fowler, \& W. K. Honig (Eds.), Cognitive processes in animal behavior. Hillsdale, N.J: Erlbaum, 1978.

Hearst, E., \& Jenkins, H. M. Sign-tracking: The stimulusreinforcer relation and directed action. Austin, Texas: Psychonomic Society, 1974.

Jenkins, H. M. Noticing and responding in a discrimination based on a distinguishing element. Learning and Motivation, 1973, 4, 115-137.

Jenkins, H. M., \& Sainsbury, R. S. The development of stimulus control through differential reinforcement. In N. J. Mackintosh \& W. K. Honig (Eds.), Fundamental issues in associative learning. Halifax: Dalhousie University Press, 1969.

Jenkins, H. M., \& Sainsbury, R. S. Discrimination learning with the distinctive feature on positive or negative trials. In D. Mostofsky (Ed.), Attention: Contemporary theory and analysis. New York: Appleton-Century-Crofts, 1970.

LEA, S. E. G. The non-occurrence of a stimulus as a signal. Quarterly Journal of Experimental Psychology, 1974, 26, 616-621.

Locurto, C., Terrace, H. S., \& Gibbon, J. Auto-shaping, random control, and omission training in the rat. Journal of Experimental Analysis of Behavior, 1976, 26, 451-462.

Peterson, G. B. Response selection properties of food and brainstimulation reinforcers in rats. Physiology \& Behavior, 1975, 14, 681-688.

Peterson, G. B., Ackil, J., Frommer, G., \& Hearst, E. Conditioned approach and contact behaviors toward signals for food or brain-stimulation reinforcement. Science, 1972, 177, 1009-1011.

SAinsbury, R. S. Effect of proximity of elements on the featurepositive effect. Journal of the Experimental Analysis of Behavior, 1971, 16, 315-325. (a)

Sainsbury, R. S. The "feature positive effect" and simultaneous discrimination learning. Journal of Experimental Child Psychology, 1971, 11, 347-356. (b)

Stiers, M., \& Silberberg, A. Lever-contact responses in rats: Automaintenance with and without a negative responsereinforcer dependency. Journal of the Experimental Analysis of Behavior, 1974, 22, 497-506.

(Received for publication July 27, 1978; revision accepted October 31,1978 .) 